\title{
Accent phrases and brain waves
}

Philippe Martin

LLF, UMR 7110, UFR Linguistique, Université Paris Diderot, France

https://doi.org/10.36505/ExLing-2017/08/0002/000304

\begin{abstract}
Recent experiments show that theta and delta brain oscillations whose periods vary from $100 \mathrm{~ms}$ to $250 \mathrm{~ms}$ and from $250 \mathrm{~ms}$ to $1300 \mathrm{~ms}$ are respectively synchronized in phase with syllables and stressed syllables. This means that, in natural speech, syllables cannot be separated by less than $100 \mathrm{~ms}$ and more than $250 \mathrm{~ms}$ to be perceived as unstressed. Over this limit, they are perceived as stressed but their separation cannot exceed $1300 \mathrm{~ms}$. Considering the brain predictive function as observed in silent reading or self-talk, where restored syllables and stressed syllables activate theta and delta oscillations, a general language acquisition and speech recognition model is suggested.
\end{abstract}

Key words: Intonation, brain oscillations, delta, theta

\section{Introduction}

Neurolinguistics is becoming one of the fastest changing domain in linguistics. After the syntactic and semantic domains, it now focuses on various aspects of speech generation, and in particular on specific speech events timing. Relationships between the perception of syllables, words or group of words boundaries and brain waves are now being investigated thoroughly in many research projects. In this contribution, I will evoke the intriguing similarities existing between stressed syllables timing and delta brain waves, leading to a model of speech processing where syllables, stressed syllables and accent phrases temporal properties are shown to correspond to those of theta, delta and gamma brain oscillations.

\section{Brain waves}

In the years 1930-40, researchers observed that the human brain consisted of a very large number of neurons (in the order of 100 billion) interconnected in groups in specific regions of the brain mass. These interconnections allow a transfer of chemically stored information in each neuron or groups of neurons by variations of a small electric potential (in the $\mu \mathrm{V}$ range). The electrical variations can be observed through captors positioned on subject's skull (electroencephalography, or EEG). These electrical variations are called evoked potential if they result from an external sensory stimulation, auditory, visual or other.

Electrical activity produced by transfers of group of neurons to other groups of neurons is not done haphazardly. First, they operate in specific

ExLing 2017: Proceedings of 8th Tutorial and Research Workshop on Experimental Linguistics, 19-22 June, Heraklion, Crete, Greece 
frequency ranges linked to specific cognitive activities, and secondly, they may synchronize in phase other waves in a different frequency range.

Greek letters designate specific frequency ranges: alpha, beta, delta, gamma. The frequency ranges of interest for speech are $1 \mathrm{~Hz}$ to $4 \mathrm{~Hz}$ for delta, $4 \mathrm{~Hz}$ to $10 \mathrm{~Hz}$ for theta, and $30 \mathrm{~Hz}$ to $80 \mathrm{~Hz}$ for gamma. The $12 \mathrm{~Hz}$ to $30 \mathrm{~Hz}$ range occupied by beta waves is also to consider. Since speech is essentially a temporal event, it may be more meaningful to characterize brain waves by their range of period variation, with delta periods varying from 250 $\mathrm{ms}$ to $1300 \mathrm{~ms}$, theta $125 \mathrm{~ms}$ to $250 \mathrm{~ms}$ and gamma $12 \mathrm{~ms}$ to $33 \mathrm{~ms}$.

The frequency of brain waves oscillations depends on the return distance from one neuronal region to another, so that the exact range of periods can vary from one subject to another. This transfer is done through a chain of synapses, whose individual length is about only 20 to $40 \mathrm{~nm}$ on the average.

Transfers between neuronal zones can be observed with a relatively large number of captors (from 32 to 256) placed around the subject skull according to location standards. Captured signals are stored in real time and analyzed into the frequency domain with either a (Fast) Fourier or Wavelet transform. The resulting representation is very similar to spectra obtained in frequency speech analysis, but in a lower frequency range.

Is then possible to observe that specific neuronal exchanges triggered by selected stimuli ("Event Related Potential", ERP) are phase coupled, with delta being the master. It has been shown for instance (Ghitza and Greenberg, 2009) that syllable peaks do synchronize theta brain waves in phase, whereas stressed syllable synchronize delta waves (Martin, 2015). Likewise, some specific frequency part of syllables, like fricative noise, synchronize gamma waves. In the absence of external stimuli, all oscillations oscillate freely in their own frequency range, delta with an at rest period of about $500 \mathrm{~ms}$, and theta with an at rest period of about $125 \mathrm{~ms}$.

\section{Designing speech with available brain waves}

Transfer of neuronal activity is essential for memorization, and some regions of the brain become specialized in information storage. Without memorization process, the image of an external sound stimulus is normally lost after 10 or 20 seconds. The acquisition of language requires thus some mechanism to encode and store the acoustic information provided by the speaker, for instance a mother to her child. The repetition of similar stimuli reinforces the neuronal memorization process, which has to be carried by some of the available brain waves.

As stressed syllables are the syllabic kernel of any word, or rather of any accent phrase (i.e. a group of syllables with one stressed syllables), a good candidate among brain waves would be delta, which would then phase lock theta oscillations while triggered by syllables (stressed or not). Gamma 
oscillations have a supplementary phase lock role for the alignment of syllables.

\section{Some experimental facts}

It is relatively easy to consider theta as responsible for the syllabic information transfer. Slowing down natural speech without modifying its spectral content down to a $100 \mathrm{~ms}$ average syllabic duration will maintain its intelligibility. Going beyond this limit would make speech unintelligible, unless some silent gap is added to each syllable to reach the $100 \mathrm{~ms}$ average duration (Ghitza and Greenberg, 2009). Slowing down speech over a $250 \mathrm{~ms}$ average syllabic duration would on the other hand make every syllable perceived as stressed, although nothing has been changed in its spectral content.

These temporal limits suggest that to be perceived, syllables must have a duration over $100 \mathrm{~ms}$ and below $250 \mathrm{~ms}$. Over $250 \mathrm{~ms}$ they are perceived as stressed. This strongly suggests that theta oscillations are carriers of nonstressed syllables. Over this $250 \mathrm{~ms}$ threshold, they will be handled by delta oscillations.

Likewise, it is relatively simple to show that stressed syllables must be at least separated by $250 \mathrm{~ms}$ by selecting two consecutive stressed syllables in natural speech and by reducing their gap, normally filled by a pause, without modifying their relative spectra. Once their separation goes below $250 \mathrm{~ms}$ the first syllable ceased to be perceived as stressed. On the other hand, two consecutive stressed syllables appear to be impossible to separate in natural speech by more than some $1300 \mathrm{~ms}$ (Martin, 2014). These two observations suggest that delta is the candidate carrier for stressed syllables.

\section{Building the lexicon}

A possible model for early language acquisition would then involve the elaboration in early childhood of a lexicon whose entries are activated by delta waves triggered by stressed syllables (including isolated syllables). Isolated vowels would progressively by replaced by full syllables, then by groups of syllables with one stressed syllable (i.e. an accent phrase), then later by sequences of accent phrases forming sentences, and eventually larger speech units involving more than one sentences (when leaning poems by hart for instance).

The various units in this lexicon are used to identify incoming speech by comparison with an existing entry, at one or more appropriate levels (syllabic, accent phrase, syntagms, etc.). Delta is the master of this continuous pattern matching operation, which requires at least $250 \mathrm{~ms}$ to be 
executed. If two stressed syllables occur at less than $250 \mathrm{~ms}$ apart, the first delta cycle is aborted and only the second one is executed.

\section{Predictive function of the brain}

The pattern matching model gives also some account for the predictive function of the brain (Arnal et Giraud, 2016). Listeners sometimes perceive stressed syllables although nothing in the speech signal carries the corresponding acoustic information. This happens when the pattern matching process found an entry in the listener lexicon that would reasonable match the speech input, restoring the stress information missing in the speech signal.

It gives also a reasonable explanation pertaining to the continuous adjustment of the delta periods according to the stressed syllables speech input. The natural oscillation normally cycles by retaking the last period value for the next, a process whose effects can be observed in eurhythmicity (Martin, 2015). However, when a stressed syllable occurs before the end of the expected period, the delta oscillation phase is reset and a new period starts. When it occurs after, the predicted period duration inferred from the lexicon retrieved entry will set the start of the new period.

This may explain why theta and delta oscillations are restored even in the absence of any speech input when reading or taking to oneself silently, their respective cycle duration being restored from the lexicon.

\section{References}

Arnal, L.H., Giraud, A-L., M. 2016. Neurophysiologie de la perception de la parole et multisensorialité, in Traité de neurolinguistique, De Boeck, Louvain-la-Neuve, $416 \mathrm{p}$.

Ghitza O" Greenberg S. 2009. On the possible role of brain rhythms in speech perception: intelligibility of time-compressed speech with periodic and aperiodic insertions of silence, Phonetica. 2009, 66(1-2), 113-26.

Martin, Ph. 2014. Spontaneous speech corpus data validates prosodic constraints, Proceedings of the 6th conference on speech prosody. Campbell, Gibbon, and Hirst (eds.), 525-529.

Martin, Ph. 2015. The Structure of Spoken Language. Cambridge University Press. 